\title{
Reflections on the Use of Perampanel in Epilepsy - Lessons from the Clinic and Real-world Evidence
}

\author{
Eugen Trinka' and Mar Carreňo² \\ 1. Department of Neurology, Christian Doppler Klinik, Paracelsus Medical University and Centre for Cognitive Neuroscience, Salzburg, Austria; \\ 2. Epilepsy Unit, Hospital Clinic, Barcelona, Spain
}

DOI: https://doi.org/10.17925/ENR.2017.12.01.17

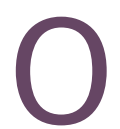

ptimal epilepsy management includes five important elements: rational treatment selection, efficacy, off-target effects, adherence and interactions and dosing issues. Perampanel (2-[2-oxo-1-phenyl-5-pyridin-2-yl-1,2-dihydropyridin-3-yl]benzonitrile; E2007) is the first potent, selective, orally-active non-competitive alpha-amino-3-hydroxy-5-methyl-4-isoxazolepropionic acid (AMPA) receptor antagonist approved for the treatment of patients with epilepsy. Results from randomised controlled trials and real-world studies of refractory epilepsy populations treated with perampanel showed effective frequency reduction for both focal-onset seizures (without and with secondary generalisation) and for primary generalised tonic-clonic seizures. Perampanel therapeutic doses have been calculated to only inhibit a fraction of AMPA receptors, thereby to enable sufficient seizure control without substantial impairment of neurological function. Further investigation in special subpopulations of people with epilepsy, including the elderly and people with learning disability or psychiatric comorbidities, is warranted. With an average long half-life of 105 hours, perampanel may be more forgiving in circumstances of suboptimal adherence. Perampanel is not a strong inducer or inhibitor of cytochrome P450 enzymes, and dose adjustment is not always required for the elderly or for those with mild renal impairment.

\section{Keywords}

AMPA receptor, anti-epileptic drugs (AEDs), real-world data, cognitive impairment, psychiatric comorbidity

Disclosure: Professor Eugen Trinka is a paid consultant for UCB, Eisai, Bial, Medtronic, EVER Neuro Pharma, Biogen-Idec, Sanofi-Genzyme, Shire, Marinus, Takeda, Newbridge and Sunovion. Professor Trinka has received research funding (directly, or to institution) from GlaxoSmithKline Biogen-Idec, Eisai, Novartis, Red Bull, Bayer, and UCB Pharma Ltd and speaker's honoraria from GlaxoSmithKline, Boehringer Ingelheim, Eisai, Bial, UCB Pharma Ltd, Sanofi-Genzyme, Shire and Sanofi-Aventis. He is the chief executive officer of Neuroconsult $\mathrm{GmbH}$ and has been awarded grants from the Austrian Science Fund (FWF), Österreichische Nationalbank, European Union. Dr Mar Carreňo has received advisory board or speaker's honoraria from Shire, Bial, Eisai, Esteve, and UCB Pharma Ltd and has received research grants from Bial and Eisai. There were no publication fees associated with the publication of this article.

Acknowledgments: Medical writing support, including preparation of the drafts under the guidance of the authors, was provided by Catherine Amey, Touch Medical Media.

Compliance with Ethics: This study involves a review of the literature and did not involve any studies with human or animal subjects performed by any of the authors.

Authorship: All named authors meet the International Committee of Medical Journal Editors (ICMJE) criteria for authorship of this manuscript, take responsibility for the integrity of the work as a whole, and have given final approval to the version to be published.

Open Access: This article is published under the Creative Commons Attribution Noncommercial License, which permits any non-commercial use, distribution, adaptation and reproduction provided the original author(s) and source are given appropriate credit. Received: 2 March 2017

Accepted: 4 March 2017

Citation: European Neurological Review, 2017;12(1):17-23

Corresponding Author: Mar Carreňo, Department of Neurology, Hospita Clínic. c/Villarroel 170, 08036, Barcelona, Spain. E: mcarreno@clinic.cat

Support: The publication of this article was supported by Eisai. The views and opinions expressed in the article are those of the authors and not necessarily those of Eisai.
As the armamentarium of anti-epileptic drugs (AEDS) continues to expand, epilepsy management is becoming increasingly complex. This necessitates multiple considerations for the choice of the most appropriate AED that can broadly be organised into five categories: (i) rational treatment selection (taking into account mode of action of AEDS); (ii) efficacy with respect to seizure control according to the patient's expectations and needs (and taking into account the seizure type(s) and syndromes); (iii) off-target effects, whereby an AED interacts with a system other than that for which it is intended (may be beneficial, for example, facilitating sleep, or harmful such as inducing dyskinesias); (iv) adherence concerns, which may involve taking into account drug characteristics, including pharmacokinetics and administration; and (v) interactions and dosing

Perampanel (2-[2-oxo-1-phenyl-5-pyridin-2-yl-1,2-dihydropyridin-3-yl] benzonitrile; E2007) is the first potent, selective, orally-active noncompetitive AMPA receptor antagonist approved for treatment of patients with epilepsy. Perampanel is indicated as an adjunctive therapy for the treatment of patients with focal-onset seizures, with or without secondarily generalised seizures, in patients with epilepsy aged 12 years or older. More recently, the European Commission approved an indication expansion for the adjunctive treatment for primary generalised tonic-clonic (PGTC) seizures in patients with idiopathic generalised epilepsy (IGE) who are at least 12 years of age. ${ }^{1}$ This review will examine these five considerations for epilepsy management as a treatment selection framework and will explore to what extent perampanel fulfils these requirements. For this purpose, the work is based on three symposia, initiated and funded by Eisai Europe, Ltd, and held at the European Congress on Epileptology (ECE), which took place in Prague, Czech Republic from 11-15 September 2016. 
Table 1: Perampanel - anti-seizure activity demonstrated in animal models

\begin{tabular}{|l|l|l|}
\hline Animal model & Potential human correlate & $\begin{array}{l}\text { Perampanel } \\
\text { anti-seizure } \\
\text { effects? }\end{array}$ \\
\hline Audiogenic (mice) & Generalised & Yes \\
\hline MES-induced (mice) & Generalised & Yes \\
\hline PTZ-induced (mice) & Absence/myoclonic & Yes \\
\hline Amygdala-kindling (rat) & Focal-onset (temporal lobe epilepsy) & Yes \\
\hline $\begin{array}{l}\text { 6 Hz electroshock (mice) } \\
32 \text { and } 44 \text { mA }\end{array}$ & Unknown & Yes \\
\hline GAERS & Absence epilepsy & No \\
\hline
\end{tabular}

Effects in animal models cannot be extrapolated to predict efficacy in humans. Perampanel is licenced for adjunctive treatment of focal-onset seizures and of primary generalised tonic-clonic seizures in idiopathic generalised epilepsy, in patients aged $\geq 12$ years. ${ }^{1}$

GAERS = genetic absence epilepsy rat from Strasbourg; MES = maximal electroshock

PTZ = pentylenetetrazol. Reproduced with permission from Walker.

\section{Rational treatment selection}

A good understanding of AED mechanisms of action (MOAs) may facilitate decision-making on the most appropriate AED or AED combination for an individual patient.

\section{Role of the AMPA receptor in epilepsy and the mode of action of perampanel}

Targeting the alpha-amino-3-hydroxy-5-methyl-4-isoxazolepropionic acid (AMPA) receptors for treatment of patients with epilepsy has generated considerable interest over the past few decades. An epileptic seizure is characterised by sudden disruption of the brain's normal electrical activity. Neurotransmitters are released when action potentials arrive at the pre-synaptic neuron ${ }^{2}$ opening voltage-gated calcium ions channels and allowing calcium ion influx. Calcium ions trigger exocytosis, releasing transmitter from vesicles into the synapse. Transmitter molecules bind to post-synaptic receptors, activating them and generating excitatory post-synaptic potentials (EPSPS). If sufficient EPSPS are triggered, the post-synaptic neuron is activated and action potentials occur. Synchronous EPSPs in groups of neighbouring neurons are responsible for epileptic field potentials. ${ }^{3}$

Glutamate is the principal excitatory neurotransmitter in the brain and glutamate-mediated excitatory neurotransmission is known to be critical in the pathophysiology of epilepsy.3.4 There are three families of glutamatergic ionotropic receptors with intrinsic cation permeable channels (N-methyl-D-aspartate [NMDA], AMPA and kainate). ${ }^{5}$ Glutamate, via the AMPA receptor, drives fast synaptic excitation at individual synapses, and across networks, whereas NMDA receptors are involved in synaptic plasticity and long-term potentiation induction. AMPA receptor antagonists, in contrast to NMDA receptor antagonists, are not known to impact synaptic plasticity, long-term potentiation and memory. ${ }^{2}$

The AMPA receptor is the predominant mediator of excitatory neurotransmission in the central nervous system (CNS). These receptors are mainly located post-synaptically and are critical to the generation and spread of epileptic activity. ${ }^{2}$ There are several lines of evidence to support the key role of the AMPA receptor in epilepsy. In early development, calcium-permeable AMPA receptors prevail and can be involved in increasing cellular calcium ion concentrations and subsequently neurotoxicity in animal models of epilepsy. ${ }^{6}$ AMPA and NMDA receptors play different roles during epileptiform activity in vitro.? Blocking NMDA receptors does not eliminate the epileptiform bursting the later bursts are inhibited but the discharge can still be triggered. By contrast, blocking AMPA receptors eliminates the epileptiform activity altogether. Perampanel has shown anti-epileptic activity in different animal models of epilepsy (Table 1), binding even when glutamate levels are high owing to its non-competitive binding properties. ${ }^{8}$

\section{Example of the involvement of AMPA receptors: focal seizures associated with brain tumours}

Focal seizures with or without secondary generalisation, are the most common symptom of brain tumours; $30-50 \%$ of these patients present with seizures; and $10-30 \%$ develop seizures later. Symptomatic management is essentially the same as for focal seizures, on the assumption that a focal brain lesion is responsible. ${ }^{10}$ Seizures associated with primary brain tumours are difficult to treat and often drug resistant; in a large cohort study, complete seizure control was achieved in 20 of 158 (12.6\%) patients with a brain tumour.11

Impaired glutamate homeostasis in and around tumours is central to seizure generation. ${ }^{2}$ Gliomas release glutamate, which has been shown to induce epileptiform activity in mice..$^{13}$ Moreover, in human glioma samples, peri-tumoural glutamate levels correlate with post-operative seizure recurrence. ${ }^{14}$ AEDS targeting the glutamate system may therefore have potential for seizure management. Electrophysiological recordings in brain slices from nine adults who underwent glioma resection showed spontaneous inter-ictal discharges; perampanel reduced the frequency of discharges, and eliminated them at higher concentrations. ${ }^{15}$ Further, the power of elicited ictal events was significantly reduced by perampanel. Perampanel is a treatment option for focal seizures associated with brain tumours; its efficacy in this setting has been demonstrated in case studies (Rosche et al. ${ }^{16}$ and data not shown) although in phase III studies of add-on perampanel in focal seizures, patients with progressive CNS tumours were excluded.

\section{Rational polytherapy}

Within the concept of 'rational polytherapy' it is thought that combining AEDs with different MOAs should be more effective than combining treatments based on the same mechanism. In theory, this approach covers multiple targets without risking additive adverse events (AEs). ${ }^{17}$ Indeed, in a real-world setting $(n=8,615)$, AED combinations with different MOAs were associated with greater treatment persistence (measured as the number of days from the index AED combination date to the end of the index combination, the end of enrolment, or the end of available data [31 March 2011], whichever occurred first) than using combinations with the same MOAs. ${ }^{18}$

Sodium channel blockade has been recognised as a major anticonvulsant mechanism in epilepsy. ${ }^{19}$ The majority of available AEDS mainly exert their effects through modulation of sodium or calcium channels, direct modulation of synaptic release, or enhancement of gamma-aminobutyric acid (GABA)-related mechanisms. Up to now, perampanel is the first and only approved selective and non-competitive AMPA receptor antagonist..$^{20}$ In three phase III randomised, double-blind, placebo-controlled trials of add-on perampanel in patients $(n=1,478)$ with refractory focal seizures, add-on perampanel in combination with one or more of the four most commonly co-administered AEDs (carbamazepine, valproic acid, lamotrigine, and levetiracetam), was efficient at reducing focal seizure frequency and improving responder rates compared with placebo, and was generally well tolerated..$^{21}$ In addition, some preclinical data suggest a supra-additive efficacy of the combination of perampanel with zonisamide in a chronic epilepsy rat model. ${ }^{22}$ Zonisamide modulates GABA-mediated neuronal inhibition, voltage-sensitive sodium channels and T-type calcium currents, thereby disrupting synchronised neuronal 
Figure 1: Adverse events reported as percentages of the 885 patients with available tolerability data from pooled pan-European real-world data ${ }^{32}$

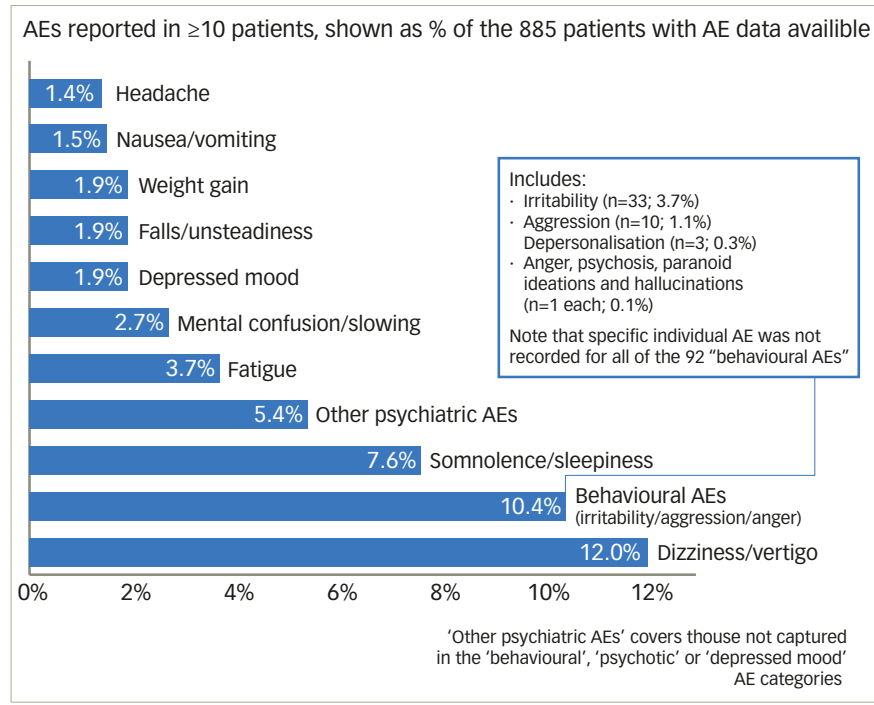

AES = adverse events. Reproduced with permission from Eugen Trinka and Georg Zimmermann.

firing, reducing the spread of seizure discharges and disrupting subsequent epileptic activity. ${ }^{23}$

The MOA of perampanel supports its use for anti-epilepsy treatment as part of rational polytherapy. However, data supporting the premise of combining drugs with different MOAs are limited to the valproic acid and lamotrigine combination ${ }^{24}$ and further investigation into this area is warranted. The concept of rational therapy remains therefore unproven as yet.

\section{Seizure control}

A crucial component of the therapeutic goal for epilepsy is to provide optimal seizure control, which meets, as far as possible, the patient's expectations and needs. Perampanel is indicated for the adjunctive treatment of patients with focal-onset seizures, with or without, secondarily generalised seizures, in adult and adolescent patients from 12 years of age with epilepsy; and for the adjunctive treatment of patients with PGTC seizures in adult and adolescent patients from 12 years of age with idiopathic generalised epilepsy. ${ }^{25}$ The efficacy and safety of perampanel have not been established for absence or myoclonic seizures, although perampanel does not seem to worsen absence/myoclonic seizures. ${ }^{1}$ The phase III clinical perampanel programme included one multicentre, double-blind, placebo-controlled study in adolescent and adults with uncontrolled PGTC seizures and IGE, ${ }^{17}$ and three multinational, double-blind, placebo-controlled studies of adjunctive perampanel (2-12 mg) in adolescents and adults with uncontrolled focal seizures despite receiving 1-3 AEDs. ${ }^{25-27}$ For the three clinical trials conducted in patients with uncontrolled focal seizures, the primary endpoint was the percentage of patients achieving at least $50 \%$ reduction in the frequency of all focal seizures per 28 days, i.e., the $50 \%$ responder rate. In pooled intent-to-treat analysis $(n=1,478)$, $50 \%$ responder rates for all focal seizures were significantly greater for perampanel compared with placebo (perampanel $4 \mathrm{mg}$, 28.5\%; $8 \mathrm{mg}$, 35.3\%; 12 mg, 35.0\%; placebo, 19.3\%; $p<0.05$, each dose versus placebo). In addition, among the patients who completed the maintenance period $(n=1,264)$, seizure-freedom rates during the maintenance period were greater with perampanel $4 \mathrm{mg}(4.4 \%), 8 \mathrm{mg}$ (3.5\%) and $12 \mathrm{mg}$ (4.1\%) than
Table 2: Most common adverse events associated with the use of perampanel in pooled Phase III trials ${ }^{21}$

\begin{tabular}{|c|c|c|c|c|}
\hline & \multirow{2}{*}{$\begin{array}{l}\text { Placebo } \\
(n=442)\end{array}$} & \multicolumn{3}{|c|}{ Perampanel } \\
\hline & & $\begin{array}{l}4 \mathrm{mg} \\
(n=172)\end{array}$ & $\begin{array}{l}8 \mathrm{mg} \\
(n=431)\end{array}$ & $\begin{array}{l}12 \mathrm{mg} \\
(n=255)\end{array}$ \\
\hline Patients with $\geq 1$ AE n (\%) & $294(67 \%)$ & $111(65 \%)$ & $350(81 \%)$ & $227(89 \%)$ \\
\hline \multicolumn{5}{|l|}{ Individual $\mathrm{AES}$ in $\geq 5 \%$ of patients } \\
\hline Dizziness & $40(9 \%)$ & $28(16 \%)$ & $137(32 \%)$ & $109(43 \%)$ \\
\hline Somnolence & $32(7 \%)$ & $16(9 \%)$ & $67(16 \%)$ & $45(18 \%)$ \\
\hline Headache & $50(11 \%)$ & $19(11 \%)$ & $49(11 \%)$ & $34(13 \%)$ \\
\hline Fatigue & $21(5 \%)$ & $13(8 \%)$ & $36(8 \%)$ & $31(12 \%)$ \\
\hline Irritability & $13(3 \%)$ & $7(4 \%)$ & $29(7 \%)$ & $30(12 \%)$ \\
\hline Nausea & $20(5 \%)$ & $5(3 \%)$ & $25(6 \%)$ & $20(8 \%)$ \\
\hline Falls & $15(3 \%)$ & $3(2 \%)$ & $22(5 \%)$ & $26(10 \%)$ \\
\hline Nasopharyngitis & $18(4 \%)$ & $9(5 \%)$ & $23(5 \%)$ & $11(4 \%)$ \\
\hline Upper respiratory tract infection & $12(3 \%)$ & $6(4 \%)$ & $14(3 \%)$ & $10(4 \%)$ \\
\hline Ataxia & $0(0 \%)$ & $1(1 \%)$ & $14(3 \%)$ & $21(8 \%)$ \\
\hline Balance disorder & $2(1 \%)$ & $0(0 \%)$ & $22(5 \%)$ & $8(3 \%)$ \\
\hline
\end{tabular}

$A E=$ adverse event

placebo (1.0\%; $p<0.05$, each dose versus placebo; seizure-freedom rate of $1.9 \%$ achieved with perampanel $2 \mathrm{mg}$ [ $p>0.05$ versus placebo]). ${ }^{2{ }^{21}}$ The mean change in frequency of secondary generalisation seizures was greater in patients receiving perampanel $2 \mathrm{mg}(-28.0 \%, \mathrm{p}=\mathrm{NS}), 4 \mathrm{mg}$ $(-48.6 \%, p<0.01), 8 \mathrm{mg}(-62.9 \%, p<0.001)$ and $12 \mathrm{mg}(-53.3 \%, p<0.001)$ than in patients receiving placebo $(-19.4 \%)$. The pooled data on efficacy and tolerability from three randomised, placebo-controlled, phase III studies of add-on perampanel in patients aged $\geq 12$ with refractory focal seizures despite taking 1-3 AEDs are presented in Table 2. The main AEs were CNS related (see Figure 1). The most frequently reported were dizziness/vertigo, behavioural AEs and somnolence/sleepiness. Most AEs were mild/moderate; relatively few patients experienced severe treatment-emergent AEs (placebo, 5.4\%; perampanel, $8.9 \%$ ) or serious treatment-emergent AEs (placebo, 5.0\%; perampanel, 5.5\%).

For the clinical trial performed in patients with uncontrolled PGTC seizures and IGE, the $50 \%$ responder rate for PGTC seizures (primary efficacy endpoint) was $64.2 \%$ for perampanel and $39.5 \%$ for placebo and the median PGTC seizure reduction was $76.5 \%$ for perampanel versus $38.4 \%$ for placebo..$^{28} \mathrm{~A}$ median daily dose of $8 \mathrm{mg}$ was achieved by 65 patients (80.2\%) treated with perampanel. PGTC seizure freedom during the maintenance phase was $30.9 \%$ for perampanel and $12.3 \%$ for placebo. To ensure enrolment of a pure population of patients with IGE, this study used an external review of every enrolled patient. The tolerability profile of perampanel shown in this study was consistent with that from studies conducted in focal seizures. ${ }^{28}$ AEs occurring in $\geq 10 \%$ with perampanel were dizziness ( $32.1 \%)$, fatigue (14.8\%), headache (12.3\%) somnolence (11.1\%), and irritability (11.1\%). Seizure control established during this core study was maintained over the course of a 144-week open-label extension phase with once-daily adjunctive perampanel up to $12 \mathrm{mg} .{ }^{29}$

Clinical trials are essential to establish safety profiles and for the approval process itself. It is important, however, to be aware of the limitations of randomised clinical trials and their implications for everyday clinical practice. Clinical records of 432 patients with epilepsy from two neurology centres in the Czech Republic were screened against the most common exclusion criteria from studies of 
Figure 2: Seizure freedom with perampanel at 12 months and last visit from pooled pan-European real-world data from 1,125 epilepsy patients ${ }^{32}$

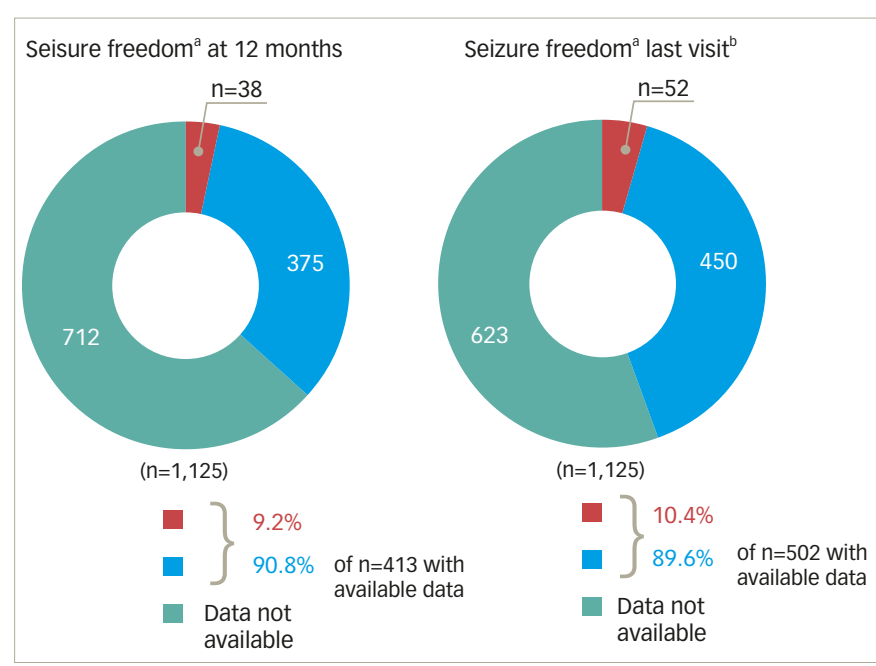

${ }^{a}$ Free of all seizures for at least the past 6 months. ${ }^{b}$ Last recorded seizure freedom data, a minimum of 6 months from perampanel initation. Reproduced with permission from Trinka and Zimmermann.

AED efficacy conducted between 2002 and 2007.30 Only 9\% of the 432 patients would have been eligible for a standard AED trial. In randomised controlled trials, syndrome-specific diagnoses are not considered, generalised epilepsies are under-represented, aetiological stratification is not acknowledged and entry information (electroencephalogram and magnetic resonance imaging, etc.) is irreversibly reduced. ${ }^{31}$ Reallife data allow information to be obtained on larger sample sizes than in randomised, controlled, clinical trials. Important insights are also gained in terms of long-term effectiveness, efficacy at different stages of treatment, patients with co-morbid conditions, and the inclusion of concomitant medication. Unusual adverse reactions may only be detected when a large population is exposed, and, finally, populations not studied in regulatory studies (e.g., the elderly, patients with learning disabilities, hepatic or renal impairment, etc.) can be included in realworld data analysis.

A large project, the pan-European real-world experience with perampanel, is ongoing. To date, pooled data from 25 epilepsy centres in Europe were analysed with the aim of providing a large dataset reflecting perampanel clinical use, including tolerability data and information on patient subpopulations, which are typically only reported as single cases or small case series. ${ }^{32}$ Preliminary analysis has been undertaken in 1125 epilepsy patients taking add-on perampanel and final results are expected in mid-2017. In 844 patients with retention data, $61 \%$ were still receiving perampanel at 12 months (median dose, 6 $\mathrm{mg}$ ), the reasons for discontinuations are still unknown for about onethird of cases, but further study is in place to address this. Overall, realworld data on perampanel, including the seizure freedom rate, seem in line with those from the clinical trial programme (Figures 1 and 2).

Most real-world data with perampanel are available for patients with focal seizures. ${ }^{33}$ However, few treatment options are available for patients with PGTC seizures and more real-world evidence is needed. A recent retrospective, single-centre post-marketing study review of medical records includ patients with focal, secondarily generalised, PGTC, and other seizures, ${ }^{34}$ including data for 101 patients taking perampanel. The responder rate (50\% seizure frequency reduction) was 51\% overall; and $53 \%$ in PGTC seizures. Most common AEs in the overall population were sleepiness/fatigue (18\%), dizziness/falls (18\%), and behavioural problems such as aggression, irritability and mood changes (15\%). Therefore, from the limited real-world evidence available in PGTC seizures, perampanel appears to be effective and well tolerated.

\section{Efficacy and safety of perampanel in special sub-populations}

In contrast to randomised clinical trials in epilepsy, the selection of AEDs in clinical practice is highly individualised and needs to consider many factors: attitudes, age, gender, seizure spectrum activity, $A E$ profile, interactions, comorbidities, contraindications, dosing and cost.

Intellectual disability and psychiatric comorbidities are both common in people with epilepsy; approximately a quarter of people with epilepsy have intellectual disability ${ }^{35}$ and their lifetime prevalence of psychiatric comorbidities is as high as $35 \%{ }^{36}$ Unfortunately, this significant population of patients is excluded from participation in randomised, clinical trials of AEDS. ${ }^{35}$ Thus, there are no current guidelines on AED selection in people with intellectual disability and the real-world evidence is sparse. In addition, in patients with epilepsy and, in particular, in this subpopulation, it is important to understand cognitive impact of AEDs. A recent retrospective study has specifically reported the use of perampanel in patients with refractory epilepsy and learning disability and/or psychiatric comorbidity. ${ }^{37}$ Retrospective data were pooled from adult patients $(n=101)$ who had received perampanel between 1 May 2014 and 3 June 2015 in a tertiary centre in France. Outcomes in patients with intellectual disability or learning disability were similar versus those without, with no significant differences in rates of $A E s$, responder rate or withdrawals. However, no patients with intellectual disability became seizure free versus $11.1 \%$ (seven patients) of those without intellectual disability (Figures $3 A$ and $3 B$ ). Outcomes were comparable between patients with psychiatric disorders versus those without (Figure 3C).

Another population that needs to be considered are elderly patients, as they are under-represented in the clinical trials and require specific considerations with respect to AED selection. ${ }^{38}$ Efficacy and side effects might differ compared with other age groups owing to agerelated brain anatomical and electrophysiological changes; differences in predominant seizure aetiology (e.g., stroke) and often higher risk of sedation, balance disorders and impact on cognitive function. In addition, there are considerations for AED selection with respect to concomitant medications. So far there is limited real-word evidence on the use of perampanel in the elderly population. In the Salzburg prospective audit, the efficacy and tolerability of perampanel in 20 elderly patients (mean age 69.8 years) were compared with that in 65 younger patients (mean age 36.8 years). ${ }^{33}$ Over 57 months, 35\% (7/20) of elderly patients were seizure free compared with $13.8 \%(9 / 65)$ of younger patients $(p=0.009)$. In the same line, in a multicentre, retrospective observational study with an overall cohort of 464 patients with refractory focal epilepsy of whom 25 were aged $\geq 65$ years, age $\geq 65$ was a predictor of seizure freedom at 12 months. ${ }^{39}$ More real-world data in this subpopulation are needed.

\section{Off-target effects}

Off-target effects are important to consider in epilepsy management; this should include anticipating and carefully explaining possible side effects without alarming the patient. Having the opportunity to discuss any fears of medication and potential side effects may enhance levels of adherence. Such discussions should include consideration of idiosyncratic side effects such as rash, liver toxicity, and QT duration and changes, as well as common side effects and how to manage them. Regarding 
perampanel, the therapeutic doses have been calculated to only inhibit a fraction of AMPA receptors, so that its efficacy does not come at the cost of substantial impairment of neurological function. ${ }^{40} \mathrm{~A}$ review of the pooled phase III data on the safety profile of perampanel in patients (age 12 and older) with focal epilepsy with or without secondary generalisation has revealed a relatively low incidence of serious treatment-emergent AEs (5.5\%), particularly at low doses, and the majority of treatment-emergent AEs were mild or moderate in intensity. ${ }^{41}$ Common neurological AEs associated with perampanel include dizziness, somnolence, ataxia, dysarthria, balance disorder and irritability. ${ }^{1}$ The safety profile of perampanel in the phase III clinical trial programme appeared consistent with that from real-world data (Figure 1).

\section{Impact on behavioural change in epilepsy}

Epilepsy, AEDs and cognition appear to form an inseparable triad. Epilepsy is associated with a broad range of neuropsychological and psychiatric comorbidities: anxiety, depression, attention deficit hyperactivity disorder (ADHD), psychosis, panic attacks, cognitive impairment and bipolar disorder. ${ }^{42,43}$ Depression is the most frequent psychiatric comorbidity in people with epilepsy, with lifetime prevalence rates from $30-35 \%{ }^{44}$ ADHD symptoms occur in nearly $20 \%$ of adults with epilepsy and are associated with increased psychosocial morbidity and lowered quality of life. ${ }^{45}$ The prevalence of anxiety disorders and psychotic disorders in patients with medically refractory focal epilepsy is about $19 \%{ }^{46}$ and $7 \%$ of patients ${ }^{47}$ respectively. Psychiatric comorbidities become an even more pressing issue with more severe epilepsy; in one study, 198 (40\%) of 490 patients with refractory focal epilepsy had psychiatric comorbidities ${ }^{48}$ and up to $65 \%$ of temporal lobectomy surgical patients have Diagnostic and Statistical Manual of Mental Disorders (DSM) axis I psychiatric disorder, most commonly depression, anxiety and organic mood/personality disorders. ${ }^{49}$

Various AEDs, including phenobarbital, clobazam, clonazepam, vigabatrin, tiagabine, topiramate, zonisamide, levetiracetam, perampanel and brivaracetam, have all been implicated in the development or worsening of behavioural and/or psychiatric symptoms in susceptible patients, many of whom have a history of these conditions and are often receiving concomitant treatment with antidepressant, antipsychotic or anxiolytic drugs. ${ }^{50}$ For example, agitation, irritability, impulsivity, anger, hostility, aggression and violence could be associated with the use of AEDs. ${ }^{51}$ Serious or life-threatening psychiatric and behavioural adverse reactions including aggression, hostility, irritability, anger, homicidal ideation and threats have been reported in patients taking perampanel. ${ }^{52}$ There are common neurotransmitter systems and brain regions implicated in both epilepsy and aggression, including the GABA, glutamate, serotonin, dopamine, and noradrenaline systems and the hippocampus, amygdala, prefrontal cortex, anterior cingulate cortex and temporal lobes. In addition, high starting dose, rapid titration, and personal and family psychiatric history all appear to be risk factors for behavioural and/or psychiatric effects. ${ }^{53}$ Other factors may also have an impact on the risk of development of psychiatric or behavioural side effects. For example, genetic variation in dopaminergic activity is associated with the risk of psychiatric side effects with levetiracetam ${ }^{54}$ and, although personalised therapy for epilepsy has yet to be developed, this may represent an opportunity to limit such AEs when selecting AEDs. Whether these behavioural and/ or psychiatric symptoms are primarily caused by the AEDs themselves or the underlying epileptic disease is subject to debate.

Behavioural and psychiatric side effects should therefore inform use of AEDs. General management recommendations are to: avoid altogether AEDs that can worsen behavioural or psychiatric side effects
Figure 3: Real-world evidence of perampanel outcomes in patients with intellectual disability (A) learning disability (B) and psychiatric comorbidity $(\mathrm{C})^{37}$

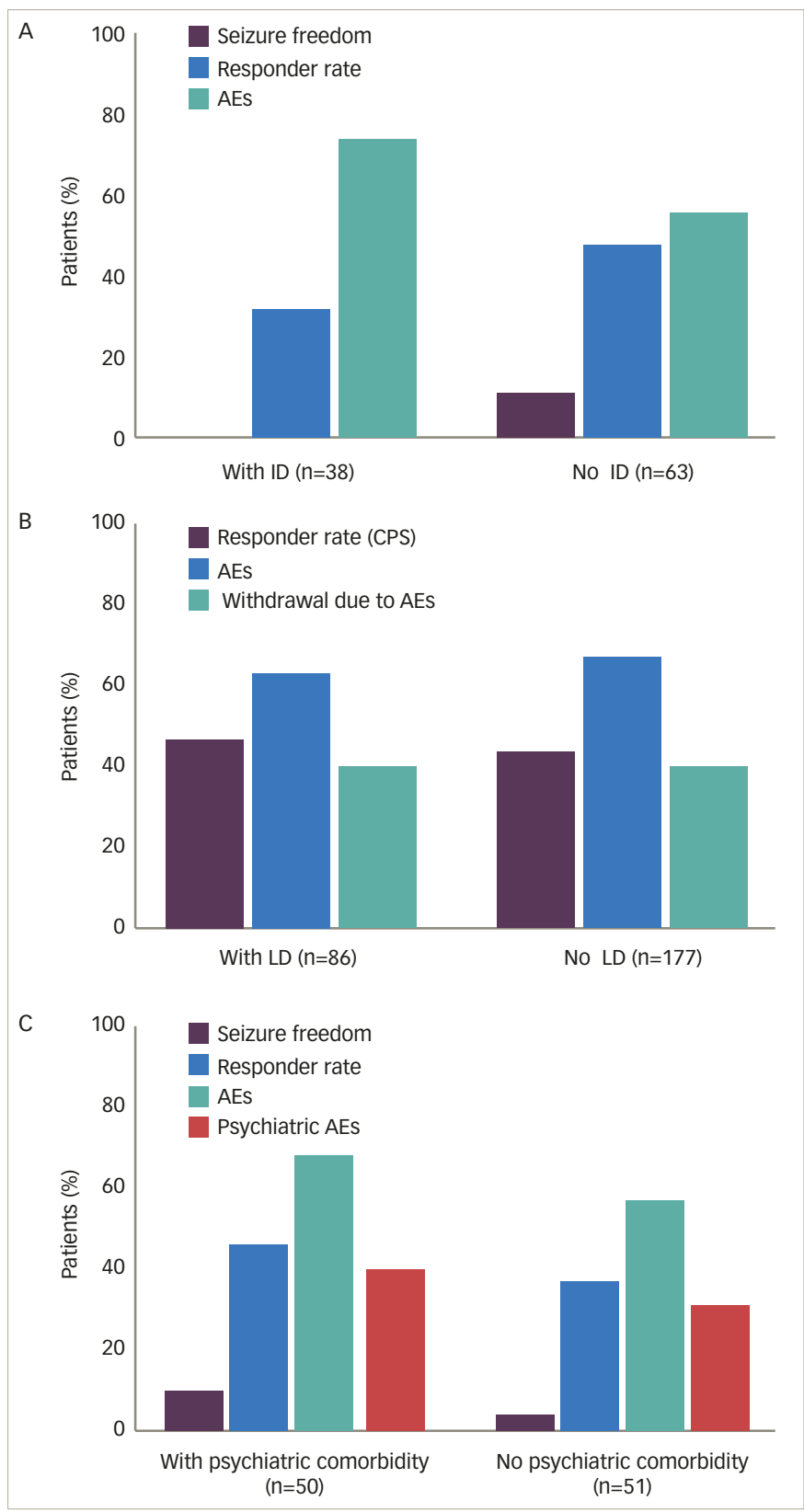

AES = adverse events; $C P S=$ complex partial seizure; ID = intellectual disability; $L D=$ learning disability.

in severely affected patients; ensure that the patient, family and general practitioner are aware of the potential for exacerbating aggression, depression, anxiety and psychosis with use of AEDs. Careful monitoring is essential. Current, past or family history of these problems should be considered, and any AED that impairs quality of life in this patient population should be replaced as early as possible, especially if they are not completely seizure free. It is advisable to titrate slowly, reduce the dose if significant problems emerge and to balance the decision to discontinue an AED with the risks of inadequate seizure control and what other AEDS are available to that patient. ${ }^{51}$

\section{Cognitive impairments and the potential impact of AEDS}

Many factors can influence cognition in epilepsy, including underlying aetiology, age at onset, seizure type and localisation of seizure, 
Table 3: Impact of anti-epileptic drugs on cognitive domains ${ }^{55}$

\begin{tabular}{|l|l|l|l|}
\hline AED & \multicolumn{3}{c|}{ Affected domains } \\
\hline Carbamazepine & Attention & Memory & \\
\hline Clobazam & $\downarrow$ & $\downarrow$ & $\downarrow$ \\
\hline Felbamate & $\downarrow$ & 0 & \\
\hline Gabapentin & $(\downarrow)$ & & 0 \\
\hline Lamotrigine & $\downarrow$ & 0 & 0 \\
\hline Levetiracetam & 0 & 0 & \\
\hline Oxcarbazepine & 0 & 0 & \\
\hline Phenobarbital & $\downarrow / \uparrow$ & $\downarrow$ & $\downarrow$ \\
\hline Phenytoin & $\downarrow$ & $\downarrow$ & \\
\hline Tiagabine & $\downarrow$ & $\downarrow$ & 0 \\
\hline Topiramate & 0 & 0 & $\downarrow$ \\
\hline Valproic acid & $\downarrow$ & $\downarrow$ & 0 \\
\hline Vigabatrin & $\downarrow$ & $\downarrow$ & 0 \\
\hline Zonisamide & 0 & 0 & $(\downarrow)$ \\
\hline & $(\downarrow)$ & & \\
\hline negative & & & \\
\hline
\end{tabular}

$\downarrow$ negative effect; $\uparrow$ positive effect; 0 possible effect; 0 no deficits; Blank, no data. AED $=$ anti-epileptic drug. Reproduced with permission from Witt and Helmstaedter, 2013.57

seizure frequency and severity, epilepsy syndrome, inter-ictal electroencephalogram abnormalities, degree of seizure control, psychosocial environment and AED treatment (Table 3). ${ }^{55}$ In a study of 247 untreated patients with newly diagnosed epilepsy, in whom the average age was 47 years, impairments in attention and executive functions were observed in $49.4 \%$ of patients and memory deficits in $47.8 \%{ }^{56} \mathrm{~A}$ review of studies in newly diagnosed and new-onset epilepsies likewise showed that cognitive deficits are already very common (14-92\%) at epilepsy onset. ${ }^{55}$ In addition, executive function has been shown to decline with increasing number of AEDS. ${ }^{57}$ In a retrospective analysis of 834 patients, total drug load was measured first, by the number of concurrent AEDS and second, as the total drug load according to defined daily dose (DDD) as provided by the World Health Organization. The cognitive measures showed higher inverse correlations with the number of AEDs (executive function: $r=-0.35, p<0.001$; memory: $r=-0.22, p<0.001)$ compared with the total DDD (executive function: $r=-0.27, p<0.001$; memory: $r=-0.17$, $p<0.001)$. A significantly lower performance in executive function was observed with each additional AED in polytherapy. In a study of 247 middle-aged patients with new onset epilepsy, impairments in memory and attention were reported in $48-49 \% .^{56}$ Studies in epilepsy surgery patients have demonstrated that AED withdrawal achieves incremental IQ gains. ${ }^{58-60}$ In a recent, randomised, placebo-controlled, phase II, double-blind study in adolescents with uncontrolled focal-onset seizures ( $n=133)$, no statistically significant difference was reported for add-on perampanel versus placebo in Cognitive Drug Research global cognition score. ${ }^{.1}$ In this study, perampanel was increased weekly in 2-mg increments to 8-12 mg/day during 6-week titration and the maintenance phase lasted 13 weeks.

The AED(s) used is only one of several influences on cognition; however, cognition can improve when seizures are controlled by AED. ${ }^{22}$ Whether cognitive impairments in patients with epilepsy are caused primarily by AEDS is therefore highly debatable but AEDS, especially in polytherapy, can worsen pre-existing deficits.

\section{Adherence}

Adherence to treatment is a critical component of epilepsy management. Non-adherence to AEDS is associated with severe clinical consequences, including increased health care utilisation and increased mortality. ${ }^{63,64} \mathrm{~A}$ relationship has been reported between poor compliance and the risk of seizures whereby every increase in daily dose frequency increased the likelihood of a seizure after a missed dose by $36 \%{ }^{65}$ Further, in an analysis of 76 studies reporting compliance measured by electronic monitoring in various disorders, the prescribed number of doses per day was inversely related to compliance: $79 \% \pm 14 \%$ if the drug was taken once daily, $69 \% \pm 15 \%$ if twice daily, $65 \% \pm 16 \%$ if three-times daily, and $51 \% \pm 20 \%$ if four-times daily. ${ }^{66}$ Many other factors influence whether patients take their medication as directed by their doctors and it is important to help optimise the probability of adherence. ${ }^{67} \mathrm{~A}$ positive therapeutic alliance will take into account patient-related factors and the factors related to the treatment, such as tolerability. This involves consideration of simple AED regimens (e.g., once daily), using educational tools or technology to help patients to remember to take their medication, facilitating patients' self-care ability and generally developing good relationships with patients so as to understand their thoughts and feelings about their medication without being judgemental.

\section{Interactions and dosing}

Many AEDS stimulate the synthesis of a wide range of monooxygenase and conjugating enzymes, which impact on the pharmacokinetics of other drugs. These include many lipid- and non-lipid-soluble drugs, including anticoagulants, cytotoxics, analgesics, antiretrovirals, glucocorticoids, statins, antihypertensives, oral contraceptives, psychoactive drugs, immunosuppressants, as well as other AEDs. Such interactions have long-term health implications including osteoporosis, sexual dysfunction and vascular disease. ${ }^{68}$ This may be a particularly relevant concern for the elderly, in whom it may be especially important for dosing and frequency considerations to fit in with patients' other drugs and routines. Perampanel is not a strong inducer or inhibitor of cytochrome P450 enzymes, and dose adjustment is not specifically recommended for the elderly or for those with mild renal impairment. ${ }^{1}$ As previously mentioned, perampanel therapeutic doses have been calculated to only inhibit a fraction of AMPA receptors. ${ }^{40}$ Further, perampanel has a half-life of approximately 105 hours so that even after abrupt treatment discontinuation, blood levels fall gradually. ${ }^{1}$

\section{Concluding remarks}

The choice of the most appropriate AED for each patient is a crucial step in epilepsy management that could take into account five important elements: rational treatment selection, seizure control, off-target effects, and adherence as well as interactions and dosing issues. The risks of inadequate seizure control should be seriously considered, as seizure control is an important determinant of injury and sudden unexpected death from epilepsy (SUDEP), but also of social and professional function. ${ }^{60}$

Perampanel is the first potent, selective, orally-active non-competitive AMPA receptor antagonist approved in the treatment of epilepsy. Overall, results in a real-life uncontrolled epilepsy population treated with perampanel showed a broadly similar level of clinical response as in the randomised controlled trials. However, more real-world data in special subpopulations of people with epilepsy, including the elderly and people with learning disability and/or psychiatric comorbidities, are needed.

The MOA of perampanel, its long half-life, together with its efficacy in both focal onset seizures and PGTC seizures in IGE, places it as an interesting option as part of rational anti-epileptic treatment. $\square$ 
1. Eisai Ltd, Fycompa, Summary of product characteristics, Available from: https://www.medicines.org.uk/emc/ medicine/26951 (accessed 20 April 2017)

2. Rogawski MA, Revisiting AMPA receptors as an antiepileptic drug target, Epilepsy Curr, 2011;11:56-63.

3. Rogawski MA, AMPA receptors as a molecular target in epilepsy therapy, Acta Neurol Scand Suppl, 2013:9-18.

4. Barker-Haliski M, White HS, Glutamatergic mechanisms associated with seizures and epilepsy, Cold Spring Harb Perspect Med, 2015;5:a022863.

5. Meldrum BS, Glutamate as a neurotransmitter in the brain: review of physiology and pathology, J Nutr, 2000;130(4S Suppl):1007s-15s.

6. Dohare P, Zia MT, Ahmed E, et al., AMPA-Kainate receptor inhibition promotes neurologic recovery in premature rabbits with intraventricular hemorrhage, I Neurosci, 2016:36:3363-77.

7. Traub RD, Miles R, Jefferys JG, Synaptic and intrinsic conductances shape picrotoxin-induced synchronized afterdischarges in the guinea-pig hippocampal slice, J Physiol, 1993;461:525-47.

8. Hanada T, Hashizume Y, Tokuhara N, et al., Perampanel: a novel, orally active, noncompetitive AMPA-receptor antagonist that reduces seizure activity in rodent models of epilepsy, Epilepsia, 2011;:52:1331-40

9. van Breemen MS, Wilms EB, Vecht CJ, Epilepsy in patients with brain tumours: epidemiology, mechanisms, and management, Lancet Neurol, 2007;6:421-30

10. Huberfeld G, Vecht CJ, Seizures and gliomas-towards a single therapeutic approach, Nat Rev Neurol, 2016;12:204-16.

11. Hildebrand J, Lecaille C, Perennes J, Delattre JY, Epileptic seizures during follow-up of patients treated for primary brain tumors, Neurology, 2005;65:212-5.

12. Pallud J, Capelle L, Huberfeld G, Tumoral epileptogenicity: how does it happen?, Epilepsia, 2013;54 Suppl 9:30-4.

13. Buckingham SC, Campbell SL, Haas BR, et al., Glutamate release by primary brain tumors induces epileptic activity, Nat Med 2011:17:1269-74.

14. Neal A, Yuen T, Bjorksten AR, et al., Peritumoural glutamate correlates with post-operative seizures in supratentoria gliomas, J Neurooncol, 2016;129:259-67.

15. Cunningham $\mathrm{M}$. Targeting elevated glutamate in brain tumour related epilepsy. Presented at: 12th European congress on Epileptology (ECE), Prague, Czech Republic, 11-15 September 2016.

16. Rosche J, Piek J, Hildebrandt G, et al., Perampanel in the treatment of a patient with glioblastoma multiforme without IDH1 mutation and without MGMT promotor methylation [Article in German], Fortschr Neurol Psychiatr, 2015;83:286-9.

17. Brodie MJ, Sills GJ, Combining antiepileptic drugs-rational polytherapy?, Seizure, 2011;20:369-75.

18. Margolis JM, Chu BC, Wang Z, et al., Effectiveness of antiepileptic drug combination therapy for partial-onset seizures based on mechanisms of action, JAMA Neurology, 2014:71:985-93.

19. Meldrum BS, Rogawski MA, Molecular targets for antiepileptic drug development, Neurotherapeutics, 2007;4:18-6

20. Hanada T, The discovery and development of perampane for the treatment of epilepsy, Expert Opin Drug Discov, 2014;9:449-58.

21. Steinhoff BJ, Ben-Menachem E, Ryvlin P, et al., Efficacy and safety of adjunctive perampanel for the treatment of refractory partial seizures: a pooled analysis of three phase III studies, Epilepsia, 2013;54:1481-9.

22. Russmann V, Salvamoser JD, Rettenbeck ML, et al., Synergism of perampanel and zonisamide in the rat amygdala kindling model of temporal lobe epilepsy, Epilepsia, 2016;57:638-47.

23. Eisai Ltd, Zonegran, Summary of product characteristics, Available from: $h$ ttp://www.ema.europa.eu/docs/en_GB/ document_library/EPAR___Product_Information/human/000577/ WC500052431 pdf (accessed 11 November 2016).

24. Moeller JJ, Rahey SR, Sadler RM, Lamotrigine-valproic acid combination therapy for medically refractory epilepsy, Epilepsia, 2009;50:475-9.
25. French JA, Krauss GL, Biton V, et al., Adjunctive perampanel for refractory partial-onset seizures: randomized phase III study 304, Neurology, 2012;79:589-96.

26. French $J A$ Krauss GL, Steinhoff BL et al Evaluation of adjunctive perampanel in patients with refractory partial-onset seizures: results of randomized global phase III study 305 , Epilepsia, 2013;54:117-25

27. Krauss GL, Perucca E, Ben-Menachem E, et al. Perampanel, a selective, noncompetitive alpha-amino-3-hydroxy-5-methyl4-isoxazolepropionic acid receptor antagonist, as adjunctive therapy for refractory partial-onset seizures: interim results from phase III, extension study 307, Epilepsia, 2013;54:126-34.

28. French JA, Krauss GL, Wechsler RT, et al., Perampanel for tonicclonic seizures in idiopathic generalized epilepsy A randomized trial, Neurology, 2015;85:950-7.

29. Wechsler R, French J, Trinka E, et al., Long-term safety and efficacy of adjunctive perampanel in patients with Wrictic in idiopathic generalised epilepsy: results of an open-label extension. Presented at: 12th European Congress on Epileptology (ECE), Prague, Czech Republic 11-15 September 2016. Abstract 555 .

30. Tlusta E, Handoko KB, Majoie M, et al., Clinical relevance of patients with epilepsy included in clinical trials, Epilepsia 2008:49:1479-80

31. Ben-Menachem E, Data from regulatory studies: What do they tell? What don't they tell?, Acta Neurol Scand Suppl, 2005;181:21-5.

32. Trinka EZ, Zimmermann G, Rohracher A, et al., A. Pan-European real-world experience with perampanel: rationale, design, and preliminary data from pooled observational studies across the continent Presented at: 12th European Congress on Epileptology (ECE), Czech Republic, 11-15 September 2016 Abstract P756.

33. Trinka E, Steinhoff BJ, Nikanorova M, Brodie MJ, Perampanel for focal epilepsy: insights from early clinical experience, Acta Neurol Scand, 2016;133:160-72

34. Singh K, Shah YD, Luciano D, et al., Safety and efficacy of perampanel in children and adults with various epilepsy syndromes: A single-center postmarketing study, Epilepsy Behav, 2016;61:41-5.

35. Doran Z, Shankar R, Keezer MR, et al., Managing anti-epileptic drug treatment in adult patients with intellectual disability: a serious conundrum, Eur J Neurol, 2016;23:1152-7.

36. Kanner AM, Management of psychiatric and neurological comorbidities in epilepsy, Nat Rev Neurol, 2016;12:106-16.

37. Maurousset A, Limousin N, Praline J, et al., Adjunctive perampanel in refractory epilepsy: Experience at tertiary epilepsy care center in Tours, Epilepsy Behav, 2016;61:237-41.

38. French JA, Staley BA, AED treatment through different ages: as our brains change, should our drug choices also? Epilepsy Curr. 2012:12(Suppl 3):22-7.

39. Villanueva V, Garces M, Lopez-Gonzalez FJ, et al., Safety, efficacy and outcome-related factors of perampanel over 12 months in a real-world setting: The FYDATA study, Epilepsy Res, 2016;126:201-10

40. Rogawski MA, Hanada T, Preclinical pharmacology of perampanel, a selective non-competitive AMPA receptor antagonist, Acta Neurol Scand Suppl, 2013:19-24.

41. Rugg-Gunn F, Adverse effects and safety profile of perampane: a review of pooled data, Epilepsia, 2014;55 Suppl 1:13-5.

42. Lin JJ, Mula M, Hermann BP, Uncovering the neurobehavioural comorbidities of epilepsy over the lifespan, Lancet, 2012:380:1180-92.

43. Ettinger AB, Reed ML, Goldberg JF, Hirschfeld RM, Prevalence of bipolar symptoms in epilepsy vs other chronic health disorders, Neurology, 2005;65:535-40.

44. Kanner AM, Schachter SC, Barry JJ, et al., Depression and epilepsy: epidemiologic and neurobiologic perspectives that may explain their high comorbid occurrence, Epilepsy Behav, 2012;24:156-68.

45. Ettinger $A B$, Ottman R, Lipton RB, et al., Attention-deficit/ hyperactivity disorder symptoms in adults with self-reported epilepsy: Results from a national epidemiologic survey of epilepsy, Epilepsia, 2015:56:218-24.

46. Brandt $\mathrm{C}$ Schoendienst $\mathrm{M}$, Trentowska $\mathrm{M}$, et al., Prevalence of anxiety disorders in patients with refractory focal epilepsy-a prospective clinic based survey Epilepsy Behav 2010:17:259-63.

47. Adams SJ, O'Brien TJ, Lloyd J, et al., Neuropsychiatric morbidity in focal epilepsy, Br J Psychiatry, 2008;192:464-9.

48. Dalmagro $\mathrm{CL}$, Velasco TR, Bianchin MM, et al., Psychiatric comorbidity in refractory focal epilepsy: a study of 490 patients, Epilepsy Behav, 2012;25:593-7.

49. Glosser G, Zwil AS, Glosser DS, et al., Psychiatric aspects of temporal lobe epilepsy before and after anterior temporal lobectomy, I Neurol Neurosurg Psychiatry, 2000;68:53-8.

50. Piedad J, Rickards H, Besag FM, Cavanna AE, Beneficial and adverse psychotropic effects of antiepileptic drugs in patients with epilepsy: a summary of prevalence, underlying mechanisms and data limitations, CNS Drugs, 2012:26:319-35

51. Brodie MJ, Besag F, Ettinger AB, et al., Epilepsy, antiepileptic drugs, and aggression: an evidence-based review, Pharmaco Rev, 2016;68:563-602

52. Eisai Ltd, Fycompa, US prescribing information, Available from: https://www.accessdata.fda.gov/drugsatfda_docs/ label/2016/208277s000lbl.pdf (accessed 29 March 2017).

53. Mula M, Trimble MR, Lhatoo SD, Sander JW, Topiramate and psychiatric adverse events in patients with epilepsy, Epilepsia, 2003;44:659-63

54. Helmstaedter $C$, Mihov Y, Toliat MR, et al., Genetic variation in dopaminergic activity is associated with the risk for psychiatric side effects of levetiracetam, Epilepsia, 2013;54:36-44.

55. Witt JA, Helmstaedter $C$, Monitoring the cognitive effects of antiepileptic pharmacotherapy-approaching the individual patient, Epilepsy Behav, 2013:26:450-6.

56. Witt JA, Helmstaedter $C$, Should cognition be screened in newonset epilepsies? A study in 247 untreated patients, J Neurol, 2012;259:1727-31.

57. Witt JA, Elger $C E$, Helmstaedter $C$, Adverse cognitive effects of antiepileptic pharmacotherapy: Each additional drug matters, Eur Neuropsychopharmacol, 2015;25:1954-9.

58. Boshuisen K, van Schooneveld MM, Uiterwaal CS, et al., Intelligence quotient improves after antiepileptic drug withdrawal following pediatric epilepsy surgery, Ann Neurol, 2015;78:104-14

59. Skirrow C, Cross JH, Cormack F, et al., Long-term intellectual outcome after temporal lobe surgery in childhood, Neurology, 2011;76:1330-7

60. Helmstaedter C, Elger CE, Witt JA, The effect of quantitative and qualitative antiepileptic drug changes on cognitive recovery after epilepsy surgery, Seizure, 2016:36:63-9.

61. Meador KJ, Yang H, Pina-Garza JE, et al., Cognitive effects of adjunctive perampanel for partial-onset seizures: A randomized trial, Epilepsia, 2016;57:243-51

62. Helmstaedter $\mathrm{C}$, Witt JA, The effects of levetiracetam on cognition: a non-interventional surveillance study, Epilepsy Behav, 2008:13:642-9.

63. Faught RE, Weiner JR, Guerin A, et al., Impact of nonadherence to antiepileptic drugs on health care utilization and costs: findings from the RANSOM study, Epilepsia, 2009:50:501-9.

64. Faught $\mathrm{E}$, Adherence to antiepilepsy drug therapy, Epilepsy Behav, 2012;25:297-302.

65. Cramer JA, Glassman M, Rienzi V, The relationship between poor medication compliance and seizures, Epilepsy Behav 2002;3:338-42

66. Claxton AJ, Cramer J, Pierce C, A systematic review of the associations between dose regimens and medication compliance Clin Ther, 2001:23:1296-310.

67. Eatock J, Baker GA, Managing patient adherence and quality of life in epilepsy, Neuropsychiatr Dis Treat,

68. Brodie MJ, Mintzer S, Pack AM, et al., Enzyme induction with antiepileptic drugs: cause for concern?, Epilepsia, 2013;54:11-27. 